\title{
The compound, diallyl disulfide, enriched in garlic, prevents the progression of doxorubicin-induced nephropathy
}

\author{
San-Chi LIN ${ }^{1}$, Amarzaya CHAGNAADORJ ${ }^{2,3}$, Uyanga BAYARSENGEE ${ }^{2,4}$, Ting-Kai LEUNG ${ }^{1,5,6,7}$, \\ Chao-Wen $\mathrm{CHENG}^{2,8,9 *}$ (D)
}

\begin{abstract}
The main medicinal property of garlic is mostly attributed to its organosulfur compounds, of which the oil-soluble diallyl disulfide (DADS) is the principal component of distilled garlic oil. Nuclear factor erythroid 2-related factor 2 (Nrf2) is an important cellular regulator in response to oxidative stress. This study assessed the possible protective effect of DADS on doxorubicin (Dox)-induced nephrotoxicity and its potential regulation of the Nrf2 pathway. Treatment with DADS (200 $\mu$ M) induced heme oxygenase (HO)-1 and NADPH quinone oxidoreductase 1 (NQO-1) expression in a human proximal tubular cell line. The induction of HO-1 expression was suppressed in Nrf2-silenced cells. In an animal study, pretreatment with DADS relieved Dox-induced albuminuria, increased catalase activity, and reduced the urinary 8-hydroxy-2'-deoxyguanosine level. In addition, DADS ameliorated the severity of glomerulosclerosis and suppressed expressions of fibrotic and inflammatory gene expressions. Our data indicate that DADS, a major component of garlic, showed protective effects of preventing the progression of Dox-induced nephropathy through enhancing Nrf2-mediated antioxidant activity. DADS, a normal constituent of the human diet, merits investigation as a potential antioxidant daily food supplement against free radical-mediated chronic diseases.
\end{abstract}

Keywords: 8-Hydroxy-2'-deoxyguanosine; catalase; heme oxygenase 1; nuclear factor erythroid 2-related factor 2; organosulfur compounds.

Practical Application: DADS can be used as daily food supplement against free radical-mediated chronic diseases.

\section{Introduction}

Nuclear factor erythroid 2-related factor 2 (Nrf2) is an important regulator of cellular resistance to oxidants; it controls the basal and induced expressions of an array of antioxidant response element (ARE)-dependent genes to regulate physiological and pathophysiological outcomes of oxidant responses. Nrf2 is translocated to nuclei when it is released from its repressive cytosolic protein, Kelch-like ECH-associated protein 1 (Keap1), and activates genes encoded with various antioxidant and phase II enzymes such as heme oxygenase (HO)-1, NADPH quinone oxidoreductase 1 (NQO1) and glutathione S-transferase (GST) (Jaramillo \& Zhang, 2013; Ma, 2013). Nrf2 and its downstream genes play very critical roles against oxidative stress and inflammation of the kidneys (Aminzadeh et al., 2013; Pedruzzi et al., 2012). For instance, $\mathrm{HO}-1$, which was critically reviewed in kidney diseases, provides a host defense mechanism, protects against oxidative stress, and contributes to renal protective activity (Nath, 2006).

Garlic is often used to add flavor to recipes and dishes. Actually, garlic has been used as a medicine for centuries due to its positive effects (Block, 1985). The main medicinal property of garlic is mostly attributed to its organosulfur compounds
(OSCs). The main initial sulfur compound is allin (S-allylcysteine sulfoxide) which occurs in intact garlic bulbs. Allin is very quickly transformed into allicin (diallyl thiosulfinate) by inducing it to release the vacuolar enzyme, alliinase. Allicin is poorly soluble in water but easily transformed into oil-soluble polysulfides, mostly diallyl disulfide (DADS), but also into diallyl sulfide (DAS) and diallyl trisulfide (DATS). Concentrations of allicin solutions were determined to be $66.7 \%$ DADS, $14.6 \%$ DATS, $13.3 \%$ DAS, and $5.4 \%$ diallyl tetrasulfide, as well as higher polysulfides, like diallyl penta- and hexasulfides which can form, but concentrations are very low (Iciek et al., 2009). From spectroscopic evidences DADS could also eliminate the toxic target of trans-crotonaldehyde in mitochondria via local electron delocalization and may further be applied for anti-myocardial ischemia (Su et al., 2018). In addition, garlic and DADS activated Nrf2 pathway to induce biogenesis in mitochondria and ameliorates hypertrophy in a isoproterenol induced cardiac hypertrophy rat model (Khatua et al., 2015). DADS protected against $\mathrm{CCl}_{4}$-induced hepatotoxicity, which was related to its ability to induce antioxidant enzymes by activating Nrf2 (Lee et al., 2015).

${ }^{1}$ Department of Internal Medicine, Taipei Hospital, Ministry of Health and Welfare, New Taipei City, Taiwan

${ }^{2}$ Graduate Institute of Clinical Medicine, College of Medicine, Taipei Medical University, Taipei, Taiwan

${ }^{3}$ Department of Internal Medicine, Mongolian National University of Medical Sciences, Ulaanbaatar, Mongolia

${ }^{4}$ Department of Internal Medicine, Shastin Central Hospital in Ulaanbaatar, Ulaanbaatar, Mongolia

${ }^{5}$ Department of Radiology, Taoyuan General Hospital, Ministry of Health and Welfare, Taoyuan City, Taiwan

${ }^{6}$ Graduate Institute of Biomedical Materials and Tissue Engineering, College of Biomedical Engineering, Taipei Medical University, Taipei, Taiwan

${ }^{7}$ College of Health Care and Management, Kainan University, Taoyuan City, Taiwan

${ }^{8}$ Traditional Herbal Medicine Research Center, Taipei Medical University Hospital, Taipei Medical University, Taipei, Taiwan

${ }^{9}$ Cell Physiology and Molecular Image Research Center, Wan Fang Hospital, Taipei Medical University, Taipei, Taiwan

${ }^{\star}$ Corresponding author: ccheng@tmu.edu.tw 
DADS significantly increased glutathione and GST activities and decreased cisplastin-induced nephrotoxicity of the kidneys (Dwivedi et al., 1996). Studies reported that DADS ameliorates gentamicin-induced renal tubular damage and oxidative stress by preserving antioxidant enzymes (Pedraza-Chaverrí et al., 2003). However, it is still unknown whether DADS can prevent glomerulonephropathy and nephrotic syndrome.

Doxorubicin (Dox)-induced nephropathy has long been used as a rodent model of chronic kidney disease, and recent studies gradually proved that Dox induces disturbances to oxidant-antioxidant systems that lead to lipid peroxidation and protein oxidation, resulting in renal tissue injury (Ayla et al., 2011). Dox-induced nephropathy is similar to human focal segmental glomerulosclerosis (FSGS), which presents with increased glomerular capillary permeability, podocyte injury (Patrakka \& Tryggvason, 2009; Wiggins, 2007), and tubular atrophy that leads to nephrotic syndrome characterized by heavy proteinuria, albuminuria, hypoalbuminemia, and hyperlipidemia (Venkatesan et al., 2000). Recent studies suggested that Dox-induced nephropathy is ameliorated by activating Nrf2 signaling pathways and reducing oxidative stress through several phytochemicals (Tsai et al., 2011; Yang et al., $2013,2014)$. In the current study, we evaluated the potential of the principal component of the distilled oil of garlic, DADS, to attenuate proteinuria progression and improve renal function in Dox-induced nephropathy.

\section{Materials and methods}

\subsection{Cell culture}

A human proximal tubular cell line (HK-2) was purchased from the Bioresource Collection and Research Center (Hsinchu, Taiwan) and routinely cultured in Dulbecco's modified Eagle medium (DMEM), supplemented with $5 \%$ fetal bovine serum (FBS), insulin-transferrin-selenium, and 1\% MycoZap Plus-CL antibiotics (Lonza, Verviers, Belgium). Cells were cultured at $37^{\circ} \mathrm{C}$ in a humidified incubator containing $5 \% \mathrm{CO}_{2}$. DADS ( $80 \%$; GC grade) was purchased from Sigma-Aldrich (St Louis, MO), and dissolved in dimethyl sulfoxide at $200 \mathrm{mM}$ as the stock concentration for the in vitro study.

The scrambled constructs and two different Nrf2 short hairpin (sh)RNA reagents were obtained from the National RNAi Core Facility Platform located at the Institute of Molecular Biology/Genomic Research Center, Academia Sinica (Taipei, Taiwan), supported by the National Core Facility Program for Biotechnology Grants of the National Science Council (NSC100-2319-B-001-002). HK-2 cells harboring the sequence of Nrf2-KD1-shRNA oligonucleotide 5'-CCGGGCTCCTACTGTGATGTGAAATCTCGAGATTTC ACATCACAGTAGGAGCTTTTT-3' (TRCN0000007555) and Nrf2KD2-shRNA oligonucleotide 5'-CCGGCCGGCATTTCACTAAACA CAACTCGAGTTGTGTTTAGT GAAATGCCGGTTTTT-3' (TRCN0000007558) were used in this study. Transfected cells were selected using $1 \mu \mathrm{g} / \mathrm{mL}$ of puromycin (Invitrogen, San Diego, CA), and the knockdown efficiency was confirmed by a real-time polymerase chain reaction (PCR).

\subsection{RNA isolation, Reverse Transcription (RT), and a quantitative RT-(q)PCR}

Total RNA was extracted with the Genezol reagent (Geneaid, New Taipei City, Taiwan) according to the Direct-zol ${ }^{\text {mo }}$ RNA MiniPrep kit instructions (Zymo Research, Orange, CA). One microgram of RNA was applied to first-strand complementary (c)DNA synthesis in a single-round RT reaction with a GoScript ${ }^{\text {ty }}$ Reverse Transcriptase Synthesis Kit according to the protocol provided by the supplier (Promega, Madison, WI). Primers applied in this study are listed in the Table 1. A real-time qPCR was conducted using $1 \mu \mathrm{L}$ of the $\mathrm{RT}$ reaction mixture as the template, gene-specific primers, ROX dye, and TOOLS $2 X$ SYBR qPCR Mix (TOOLS Biotech, New Taipei City, Taiwan). Results were analyzed using the TOptical Thermocycler Real-Time PCR System (Biometra, Göttingen, Germany). Amplifications were normalized using the $2^{-\Delta \Delta C T}$ method as pervious described (Lin et al., 2018).

\subsection{Immunoblots}

For total cellular protein, cells were washed and then lysed in a PRO-PREP Protein extraction solution (iNtRON Biotechnology, Kyungki-Do, South Korea) with indicated treatments and at certain time points. For the Western blot analysis, protein lysates ( $30 \mu \mathrm{g} / \mathrm{lane}$ ) were separated by a $4-20 \%$ grading sodium dodecylsulfate polyacrylamide gel electrophoresis (SDS-PAGE) gel and electroblotted onto polyvinylidene difluoride membranes. The membranes were blocked with $0.5 \%$ bovine serum albumin (BSA) in phosphate-buffered saline (PBS) and were then incubated with HO-1 and $\beta$-actin antibodies. The bound antibodies were detected after incubation with a horseradish peroxidase-conjugated secondary antibody and were visualized with an enhanced chemiluminescence system.

\subsection{Animal study}

An experiment was conducted on 8-week-old female BALB/c mice provided by the National Laboratory Animal Center (Taipei, Taiwan). An experimental FSGS model was induced by an intravenous injection of a single dose of Dox $(10 \mathrm{mg} / \mathrm{kg}$ body weight, Sigma-Aldrich) as pervious described (Lin et al., 2018). Disease onset was checked regularly using urine dipsticks (Siemens, Tokyo, Japan). Two days before the Dox injection (recorded as day 0 for FSGS model induction), a group of mice $(n=8)$ was given a daily dose of DADS $(30 \mathrm{mg} / \mathrm{kg}$ body weight, Sigma-Aldrich) in $50 \mu \mathrm{L}$ corn oil or not $(n=8)$ throughout the experiment. Age- and sex-matched BALB/c mice $(n=6)$ were given corn oil by oral gavage and used as the normal control. Experimental mice were sacrificed at 21 days after the Dox injection. Urine samples were collected and measured once a week after disease induction until the mice were sacrificed.

\subsection{Evaluation of albuminuria}

Urine samples were diluted 50-fold in PBS, and creatinine ( $\mathrm{Cr}$ ) levels were further determined by a dry multilayer analytical slide method with the Roche Reflotron Plus Dry Chemistry Analyzer (Roche, Mannheim, Germany). Urine albumin concentrations were determined by a standard Bio-Rad Bradford protein assay. 
Table 1. Primer sequences.

\begin{tabular}{lll}
\hline & \multicolumn{1}{c}{ Forward sequence } & Reverse sequence \\
\hline Human & & \\
Heme oxygenase-1 & CCAGGCAGAGAATGCTGAGTTC & AAGACTGGGCTCTCCTTGTTGC \\
NAD $($ P H quinone dehydrogenase 1 & CCTGCCATTCTGAAAGGCTGGT & GTGGTGATGGAAAGCACTGCCT \\
Glyceraldehyde 3-phosphate dehydrogenase & GTCTCCTCTGACTTCAACAGCG & ACCACCCTGTTGCTGTAGCCAA \\
Mouse & & \\
Fibronectin & CCCTATCTCTGATACCGTTGTCC & TGCCGCAACTACTGTGATTCGG \\
TIMP metallopeptidase inhibitor 1 & TCTTGGTTCCCTGGCGTACTCT & GTGAGTGTCACTCTCCAGTTTGC \\
Monocyte chemoattractant protein-1 & CACCTGCTGCTACTCATTC & TCTGGACCCATTCCTTCTTG \\
Vascular cell adhesion molecule-1 & GCTATGAGGATGGAAGACTCTGG & ACTTGTGCAGCCACCTGAGATC \\
Glyceraldehyde 3-phosphate dehydrogenase & CATCACTGCCACCCAGAAGACTG & ATGCCAGTGAGCTTCCCGTTCAG \\
\hline
\end{tabular}

Urine albumin excretion in the same sample was expressed as the albumin-creatine ratio (ACR; $\mathrm{mg} / \mathrm{mg}$ ) as pervious described (Lin et al., 2018).

\subsection{Evaluation of renal histopathology}

Formalin-fixed and paraffin-embedded renal sections $(4 \mu \mathrm{m})$ were prepared and stained with periodic acid-Schiff (PAS) for a renal histological examination. A semiquantitative glomerular injury score was used to assess the degree of glomerular injury. The degree of glomerular injury in 50 glomeruli was graded using a scale of 0 to 4 , based on the percentage of glomerular involvement; score 0: normal; score 1: lesions in up to $25 \%$ of glomeruli; score 2: lesions in 25-50\%; score 3: lesions in 50-75\%; and score 4 : lesions in $75-100 \%$ of glomeruli. The glomerular injury score was then calculated by dividing the total score by the total number of glomeruli evaluated.

\subsection{Determination of catalase activity}

After being perfused with PBS to remove red blood cells and clots, renal cortical tissues were homogenized in $50 \mathrm{mM}$ phosphate buffer ( $\mathrm{pH}$ 7.4) with a mechanical homogenizer (Analytik Jena SpeedMill PLUS, Jena, Germany). Suspensions were centrifuged at $13,000 \mathrm{xg}$ and $4{ }^{\circ} \mathrm{C}$ for $30 \mathrm{~min}$, and the supernatants were used for the assay. Catalase activities in renal cortical tissue were measured using a Caymen Chemical kit (Ann Arbor, MI).

\subsection{Determination of urinary 8-hydroxy-2'-deoxyguanosine (8-OHdG) with an enzyme-linked immunosorbent assay (ELISA)}

Urinary concentrations of 8-OHdG were determined by a commercial competitive ELISA kit from the Japan Institute for the Control of Aging (Fukuroi, Shizuoka, Japan).

\subsection{Statistical analysis}

All results are expressed as the mean \pm standard deviation (SD). Comparisons between two groups were performed by an unpaired Student's $t$-test. Differences among multiple groups were determined by a one-way analysis of variance (ANOVA) with Tukey's method for the post-hoc analysis. $p<0.05$ was considered statistically different.

\section{Results}

\subsection{DADS induced HO-1 and NQO-1 expression}

Inasmuch as oxidative stress is a causal pathologic factor for kidney diseases, we first examined whether DADS induced Nrf2-antioxidant response element-dependent gene expressions. Transcriptional levels of HO- 1 and NQO- 1 were measured after treatment of HK-2 cells with DADS for 6 and $18 \mathrm{~h}$. Treatment with $200 \mu \mathrm{M}$ DADS rapidly induced HO-1 mRNA expression at $6 \mathrm{~h}$. Although it declined thereafter, the increase in HO-1 expression lasted to $18 \mathrm{~h}$ (Figure 1A, B) with 100 and $200 \mu \mathrm{M}$ DADS treatment. The upregulated expression of NQO-1 was only observed after treatment with $200 \mu \mathrm{M}$ DADS at $18 \mathrm{~h}$ (Figure 1C, D).

\subsection{DADS induced Nrf2 activation}

To confirm that DADS-induced HO-1 expression mediates Nrf2 activation, we evaluated DADS' effects on both HO-1 messenger (m)RNA and protein levels with two different Nrf2-silenced HK-2 cell lines. Consistently, treatment with $200 \mu \mathrm{M}$ DADS induced HO-1 expression in HK-2-scrambled cells at $6 \mathrm{~h}$, and its induction was suppressed in Nrf2-silenced cells (Figure 2A). This result was also confirmed by the induction of HO-1 protein levels (Figure 2B). This evidence suggested that DADS induced HO-1 and NQO-1 expressions through activation of $\mathrm{Nrf} 2$.

\subsection{DADS relieved Dox-induced oxidative injury}

Then, we further evaluated the preventive abilities of DADS in a mouse model of Dox-induced renal injury. As shown in Figure 3A, an intravenous injection of Dox led to significant proteinuria at 14 days after the injection which continued to day 21. Levels of urinary 8-OHDG also increased in the Dox-treated group at day 21. Pretreatment with DADS significantly ameliorated the proteinuria and reduced levels of urinary 8-OHDG (Figure 3B). In addition, DADS pretreatment also increased catalase activities in renal cortical tissues (Figure 3C). These data indicated that DADS possibly prevented Dox-induced oxidative injury through the enhancement of antioxidant abilities. 
(A)

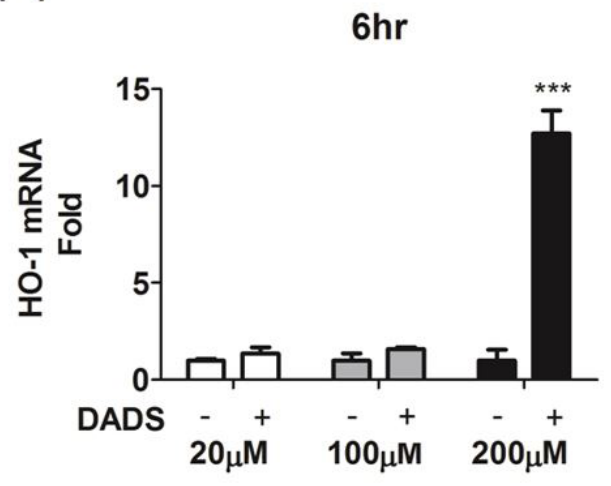

(C)

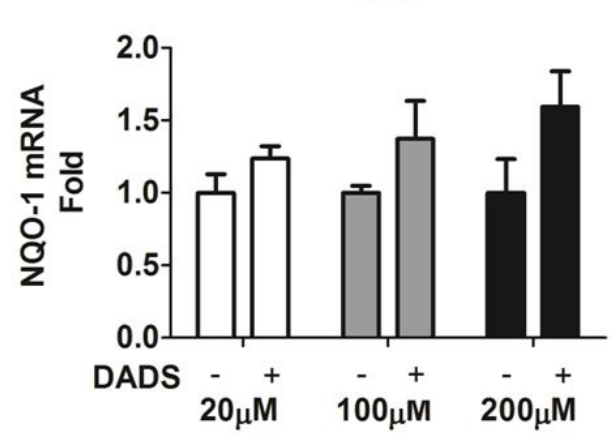

(B)

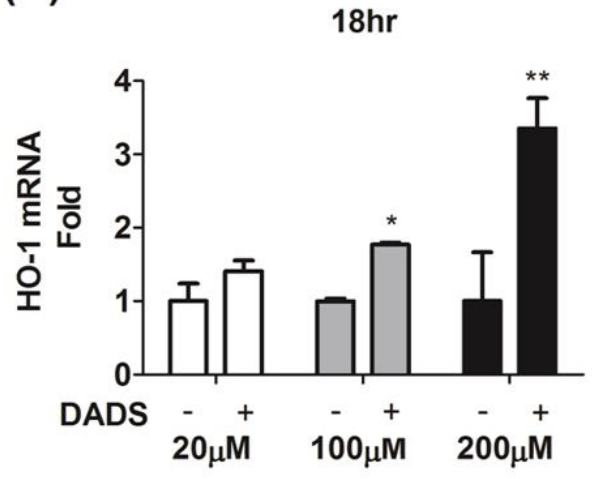

(D)

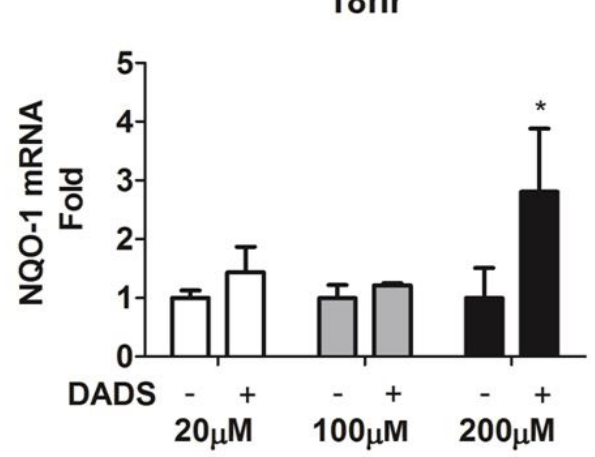

Figure 1. HO-1 mRNA expression levels with different concentrations of DADS at 6 (A) and $18 \mathrm{~h}(\mathrm{~B})$. NQO-1 mRNA expression levels with different concentrations of DADS at $6(\mathrm{C})$ and $18 \mathrm{~h}(\mathrm{D}) .{ }^{*} p<0.05 ;{ }^{* *} p<0.01$; ${ }^{* *} p<0.001$ compared to the group treated with $0 \mu \mathrm{M}$.

(A)

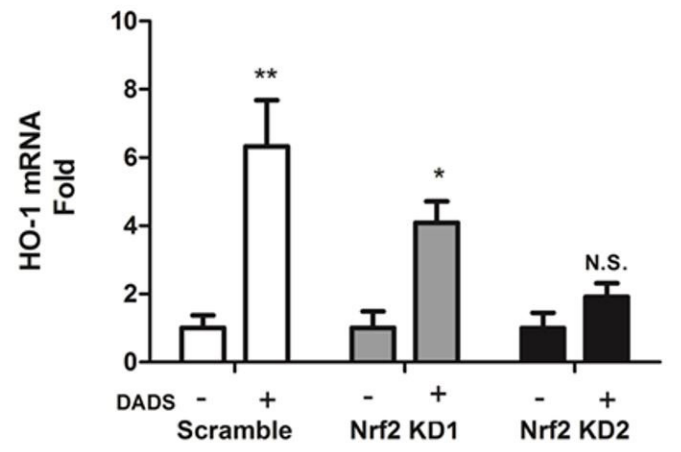

(B)

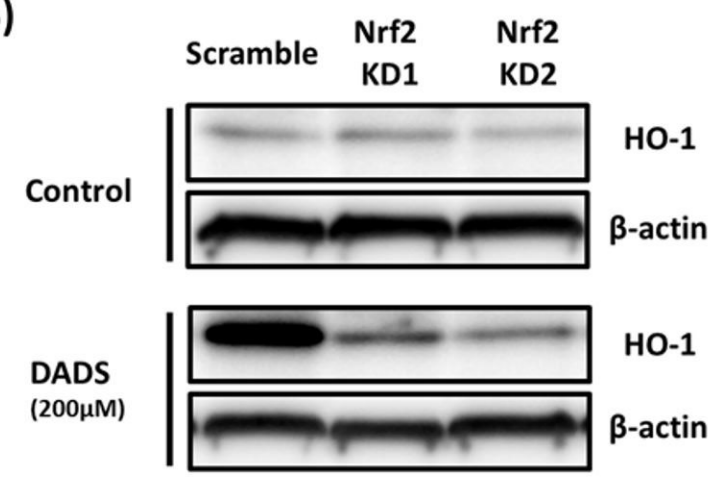

Figure 2. Relative HO-1 mRNA expressions in scrambled (Sc), Nrf2-KD1, and Nrf2-KD2 HK-2 cells upon $200 \mu$ M DADS treatment at 6 h. Expression levels are shown as relative multiples of individual unstimulated controls (A). ${ }^{*} p<0.05 ;{ }^{* *} p<0.01$ compared to the group treated with $0 \mu \mathrm{M}$; N.S. represents no significance. Representative immunoblots of HO-1 expression $(24 \mathrm{~h})$ in unstimulated and stimulated conditions in Sc, Nrf2-KD1, and Nrf2-KD2 cells; $\beta$-actin was used as the loading control (B).

\subsection{DADS prevented Dox-induced renal injury'}

Dox-treated animals showed histopathological features of segmental to global glomerulosclerosis, extracellular matrix (ECM) deposits, mesangial expansion, tubular dilation, and protein casts. Pretreatment with DADS ameliorated the sclerotic lesions in the Dox-treated group (Figure 4A, B).
Dox-induced upregulation of fibrotic genes, fibronectin, and tissue inhibitor of metalloproteinase (TIMP) was suppressed in the DADS-pretreated group (Figure 4C, D). In addition, DADS also showed activities in relieving inflammation related genes, monocyte chemoattractant protein- 1 and vascular cell adhesion molecule-1 expression (Figure 4E, F) 
(A)

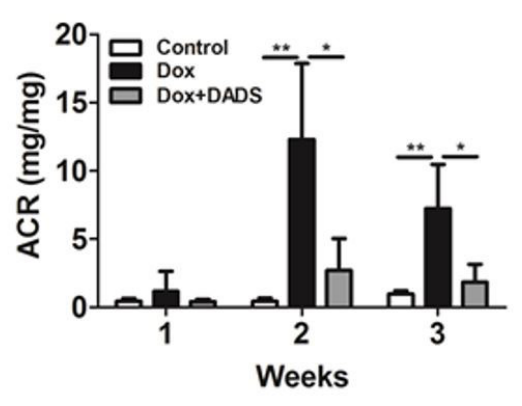

(B)

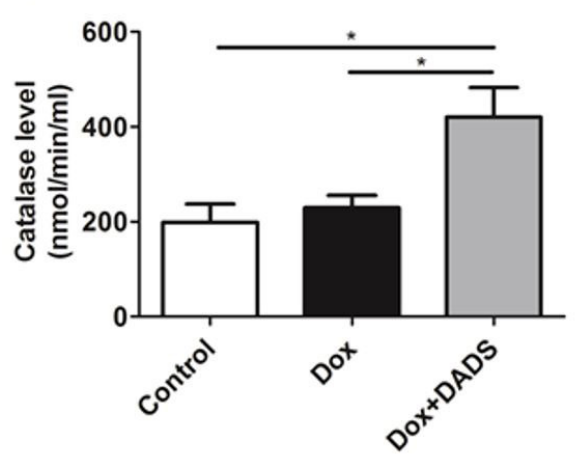

(C)

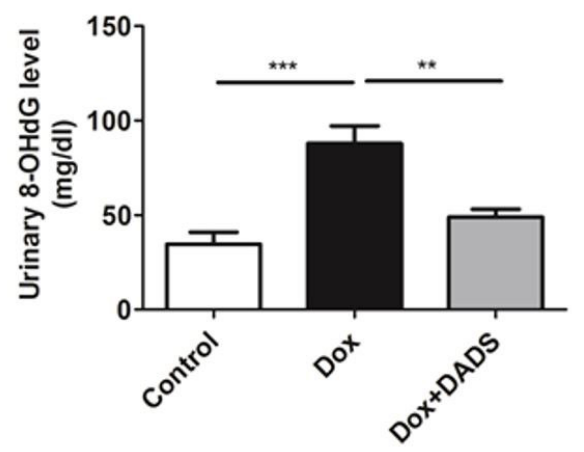

Figure 3. The urinary albumin-creatine ratio (ACR) was analyzed at 7, 14, and 21 days after Dox administration (A). At the end of the experiment, levels of tissue catalase activity (B) and urinary 8-hydroxy-2'-deoxyguanosine (8-OHDG) (C) were analyzed. ${ }^{*} p<0.05$; $^{* *} p<0.01$; $^{* * *} p<0.001$.

(A)

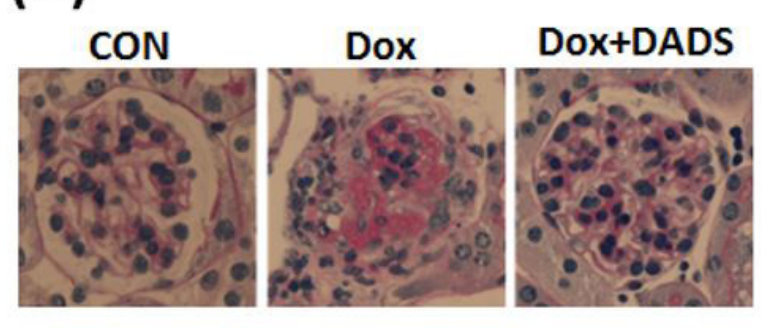

(C)

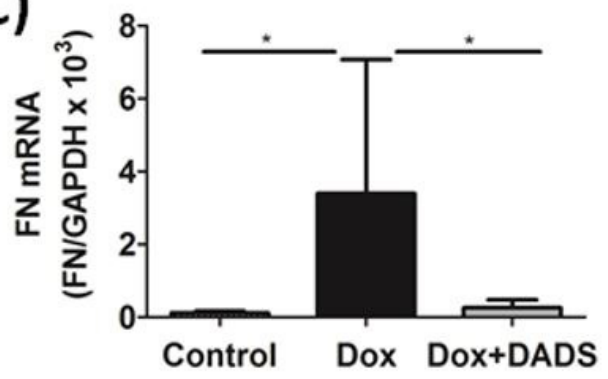

(E)

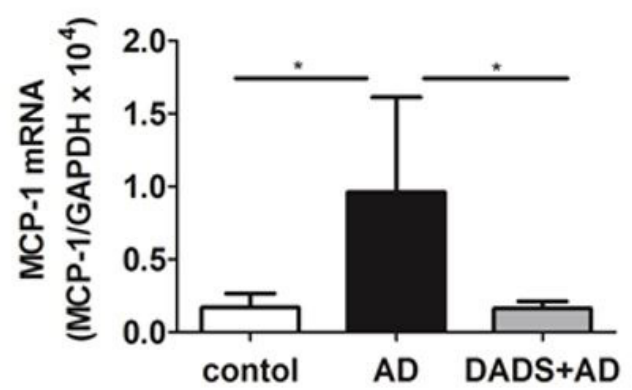

(B)

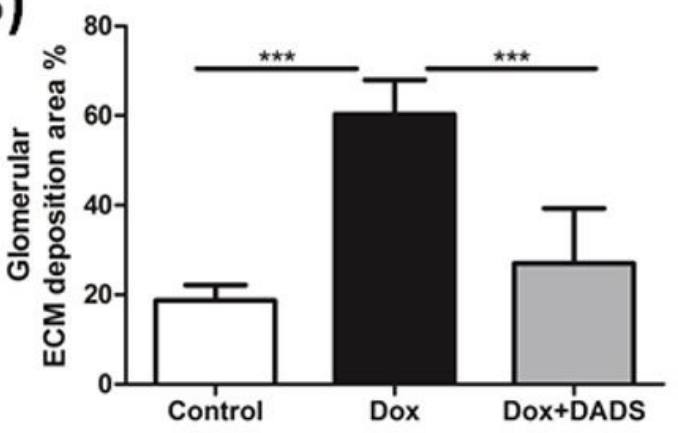

(D)

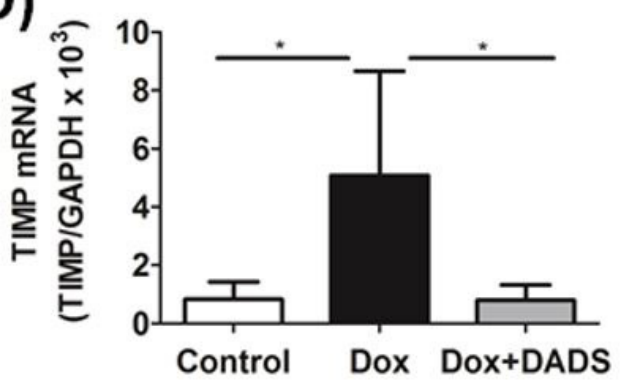

(F)

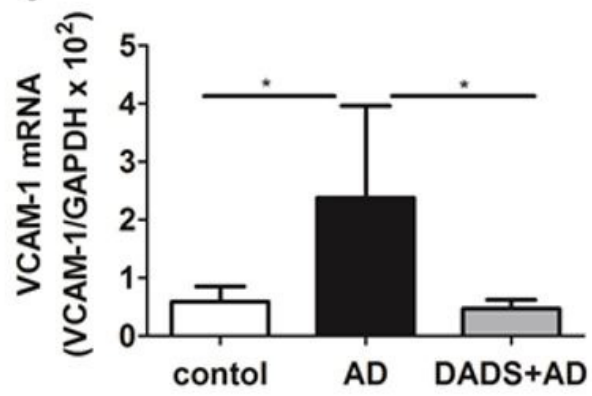

Figure 4. Representative PAS staining pictures of glomeruli in kidney samples from all groups (A), and the degrees of glomerulosclerosis were analyzed (B). Renal cortical tissue fibronectin (FN) (C), tissue inhibitor of metalloproteinase (TIMP) (D), monocyte chemoattractant protein-1 $(\mathrm{MCP}-1)(\mathrm{E})$ and vascular cell adhesion molecule-1 (VCAM-1) (F) mRNA expressions were analyzed. ${ }^{*} p<0.05 ;{ }^{* * *} p<0.001$. 


\section{Discussion}

As mentioned earlier, DADS is the major component of garlic oil, which is easy to obtain and apply to various foods. In the current study, we verified the abilities of DADS to modulate in vitro and in vivo antioxidant activities. Our data showed that DADS can induce HO-1 and NQO-1 gene expressions through Nrf2 activation in the HK-2 cell line. In addition, daily oral intake of $30 \mathrm{mg} / \mathrm{kg}$ DADS significantly ameliorated Dox-induced albuminuria and oxidative injury. Dox-induced glomerulosclerosis, fibrotic and inflammatory gene expressions were also attenuated. This evidence supports the beneficial role of DADS in preventing oxidative stress-related tissue injury.

Dox-induced nephropathy is a commonly used animal model for evaluating therapeutic effects of compounds in human nephrotic syndrome. Recently, studies proved that several phytochemicals showed activities of activating Nrf2 and ameliorated the progression of Dox-induced nephropathy. Yang et al. $(2013,2014)$ reported that citral (a major active compound of Litsea cubeba) and osthole (a coumarin compound isolated from seeds of Cnidium monnieri), can reduce glomerular epithelial hyperplastic lesions and glomerular hyalinosis/sclerosis. In addition, they suggested that they exhibited renoprotective activities through inhibiting oxidative stress and apoptosis, and causing early activation of the Nrf2 pathway. Tsai et al. (2011) also indicated that in addition to inhibiting renal nuclear factor- $\kappa \mathrm{B}$ activation and decreasing levels of transforming growth factor- $\beta 1$ in serum, antroquinonol (a major active component of Antrodia camphorate) also increased Nrf2 activation to relieve Dox-induced nephropathy. In our recent findings, the natural compound $2,3,5,4^{\prime}$-tetrahydroxystilbene-2-O- $\beta$ - $d$ glucoside (an active compound extracted from Polygonum multiflorum Thunb) also protected against Dox-induced nephropathy through activating the Nrf2-Keap1 antioxidant pathway (Lin et al., 2018). In the current study, silencing Nrf2 suppressed DADS-induced HO-1 mRNA and protein expressions, supporting the ability of DADS to activate the Nrf2 pathway. In addition, DADS also suppressed oxidative injury and Dox-induced nephropathy. This evidence supports the role of DADS in Nrf2 activation and inducing an array of ARE-dependent genes in protecting against oxidative stress-related tissue injuries.

In addition to Dox-induced nephropathy, DADS also showed activities on anti-inflammation in several different diseases models. Garlic Oil and DADS showed preventive effects on cigarette smoke-induced airway inflammation and oxidation in mice (Ko et al., 2018; Liu et al., 2018). In an ovalbumin-induced allergic asthma model, DADS also relieved airway inflammation through activating Nrf2/HO-1 pathway and inhibiting NF- $\kappa \mathrm{B}$ activation (Shin et al., 2013). Besides, oral administration of DAS and DADS also ameliorated experimental inflammatory bowel disease murine model (Fasolino et al., 2015). In the current study, preventive DADS treatment not only ameliorated the disease severity of Dox-nephropathy, but also showed activities on suppression the expression of VCAM-1 and MCP-1 (Figure 4E, F). This evidence may also highlight the potential regulatory activities of DADS in the anti-inflammation, however, the detail mechanism need further elucidation.

DATS also showed strong antioxidant activities (Fukao et al., 2004; Wu et al., 2001). Wu et al. examined the effects of garlic oil and OSOCs on the antioxidation system in the rat liver and red blood cells. Garlic oil, DADS, and DATS all increased the glutathione (GSH) content in red blood cells, while DATS showed superior activity. DADS and DATS significantly increased the activities of GSH reductase and GSH S-transferase (GST), but decreased GSH peroxidase activity in the liver (Wu et al., 2001). Kim et al. (2014) also found that both DADS and DATS can induce HO-1 and NQO-1 expressions in human gastric epithelial cells. They suggested that DATS may directly interact with the Cys 288 residue of Keap 1 to induce Nrf2 activation and upregulate defensive gene expressions. However, the underlying mechanisms by which DADS modulates Nrf2 activation still need further investigation.

In addition to oil-soluble OSOCs, water-soluble OSOCs, including the allyl amino acid derivatives S-allylcysteine and S-allylmercaptocysteine, also showed antioxidant activities in vitro and in vivo studies (Borek, 2001; Moriguchi et al., 1997). Aged garlic extract (AGE), a garlic preparation rich in water-soluble cysteinyl moieties, was also reported to have renoprotective activities. AGE protected against oxidative stress and renal changes in cisplatin- or cyclosporine-induced nephropathy (Nasr \& Saleh, 2014; Wongmekiat \& Thamprasert, 2005). In addition, both DADS and AGE were protective against cadmium (Cd)-induced toxicity through induction of cytoprotective enzymes in an Nrf2-dependent manner (Lawal \& Ellis, 2011).

\section{Conclusions}

In the current study, we found that the daily uptake of the oil-soluble OSOC, DADS, can prevent Dox-induced nephropathy through enhancing antioxidant activities. This finding further proves the benefits of daily food supplements with potent natural antioxidant activities. In conclusion, this study provided evidence supporting the health benefits of garlic for preventing environmental oxidative stress.

\section{Acknowledgements}

Funding sources for the work was supported by the Taipei Hospital, Ministry of Health and Welfare (201504).

\section{References}

Aminzadeh, M. A., Nicholas, S. B., Norris, K. C., \& Vaziri, N. D. (2013). Role of impaired Nrf2 activation in the pathogenesis of oxidative stress and inflammation in chronic tubulo-interstitial nephropathy. Nephrology, Dialysis, Transplantation, 28(8), 2038-2045. http://dx.doi. org/10.1093/ndt/gft022. PMid:23512109.

Ayla, S., Seckin, I., Tanriverdi, G., Cengiz, M., Eser, M., Soner, B., \& Oktem, G. (2011). Doxorubicin induced nephrotoxicity: protective effect of nicotinamide. International Journal of Cell Biology, 2011, 390238. http://dx.doi.org/10.1155/2011/390238. PMid:21789041.

Block, E. (1985). The chemistry of garlic and oniono. Scientific American, 252(3), 114-119. http://dx.doi.org/10.1038/scientificamerican0385-114. PMid:3975593.

Borek, C. (2001). Antioxidant health effects of aged garlic extract. The Journal of Nutrition, 131(3s), 1010S-1015S. http://dx.doi.org/10.1093/ jn/131.3.1010S. PMid:11238807.

Dwivedi, C., Abu-Ghazaleh, A., \& Guenther, J. (1996). Effects of diallyl sulfide and diallyl disulfide on cisplatin-induced changes in glutathione and glutathione-S-transferase activity. Anti-Cancer 
Drugs, 7(7), 792-794. http://dx.doi.org/10.1097/00001813-19960900000012. PMid:8949992.

Fasolino, I., Izzo, A. A., Clavel, T., Romano, B., Haller, D., \& Borrelli, F. (2015). Orally administered allyl sulfides from garlic ameliorate murine colitis. Molecular Nutrition \& Food Research, 59(3), 434442. http://dx.doi.org/10.1002/mnfr.201400347. PMid:25488545.

Fukao, T., Hosono, T., Misawa, S., Seki, T., \& Ariga, T. (2004). Chemoprotective effect of diallyl trisulfide from garlic against carbon tetrachloride-induced acute liver injury of rats. BioFactors, 21(1-4), 171-174. http://dx.doi.org/10.1002/biof.552210135. PMid:15630193.

Iciek, M., Kwiecien, I., \& Wlodek, L. (2009). Biological properties of garlic and garlic-derived organosulfur compounds. Environmental and Molecular Mutagenesis, 50(3), 247-265. http://dx.doi.org/10.1002/ em.20474. PMid:19253339.

Jaramillo, M. C., \& Zhang, D. D. (2013). The emerging role of the Nrf2-Keap1 signaling pathway in cancer. Genes \& Development, 27(20), 2179-2191. http://dx.doi.org/10.1101/gad.225680.113. PMid:24142871.

Khatua, T. N., Dinda, A. K., Putcha, U. K., \& Banerjee, S. K. (2015). Diallyl disulfide ameliorates isoproterenol induced cardiac hypertrophy activating mitochondrial biogenesis via eNOS-Nrf2-Tfam pathway in rats. Biochem Biophys Rep, 5, 77-88. PMid:28955809.

Kim, S., Lee, H.-G., Park, S.-A., Kundu, J. K., Keum, Y.-S., Cha, Y.-N., Na, H.-K., \& Surh, Y.-J. (2014). Keap1 cysteine 288 as a potential target for diallyl trisulfide-induced Nrf2 activation. PLoS One, 9(1), e85984. http://dx.doi.org/10.1371/journal.pone.0085984. PMid:24489685.

Ko, J. W., Jeong, S. H., Kwon, H. J., Shin, N. R., Seo, Y. S., Kim, J. C., Shin, I. S., \& Kim, J. S. (2018). Preventive effect of garlic oil and its organosulfur component diallyl-disulfide on cigarette smoke-induced airway inflammation in mice. Nutrients, 10(11), 1659. http://dx.doi. org/10.3390/nu10111659. PMid:30400352.

Lawal, A. O., \& Ellis, E. M. (2011). The chemopreventive effects of aged garlic extract against cadmium-induced toxicity. Environmental Toxicology and Pharmacology, 32(2), 266-274. http://dx.doi. org/10.1016/j.etap.2011.05.012. PMid:21843808.

Lee, I. C., Kim, S. H., Baek, H. S., Moon, C., Kim, S. H., Kim, Y. B., Yun, W. K., Kim, H. C., \& Kim, J. C. (2015). 2013. Protective effects of diallyl disulfide on carbon tetrachloride-induced hepatotoxicity through activation of Nrf2. Environmental Toxicology, 30(5), 538-548. http://dx.doi.org/10.1002/tox.21930. PMid:24293383.

Lin, E. Y., Bayarsengee, U., Wang, C. C., Chiang, Y. H., \& Cheng, C. W. (2018). The natural compound 2,3,5,4'-tetrahydroxystilbene-2-Obeta-d glucoside protects against adriamycin-induced nephropathy through activating the Nrf2-Keap1 antioxidant pathway. Environmental Toxicology, 33(1), 72-82. http://dx.doi.org/10.1002/tox.22496. PMid:29064158.

Liu, Y., Li, A., Feng, X., Sun, X., Zhu, X., \& Zhao, Z. (2018). Pharmacological investigation of the anti-inflammation and anti-oxidation activities of diallyl disulfide in a rat emphysema model induced by cigarette smoke extract. Nutrients, 10(1), 79. http://dx.doi.org/10.3390/ nu10010079. PMid:29329251.

Ma, Q. (2013). Role of nrf2 in oxidative stress and toxicity. Annual Review of Pharmacology and Toxicology, 53(1), 401-426. http://dx.doi. org/10.1146/annurev-pharmtox-011112-140320. PMid:23294312.

Moriguchi, T., Saito, H., \& Nishiyama, N. (1997). Anti-ageing effect of aged garlic extract in the inbred brain atrophy mouse model. Clinical and Experimental Pharmacology \& Physiology, 24(3-4), 235-242. http://dx.doi.org/10.1111/j.1440-1681.1997.tb01813.x. PMid:9131291.

Nasr, A. Y., \& Saleh, H. A. (2014). Aged garlic extract protects against oxidative stress and renal changes in cisplatin-treated adult male rats. Cancer Cell International, 14(1), 92. http://dx.doi.org/10.1186/ s12935-014-0092-x. PMid:25298749.
Nath, K. A. (2006). Heme oxygenase-1: a provenance for cytoprotective pathways in the kidney and other tissues. Kidney International, 70(3), 432-443. http://dx.doi.org/10.1038/sj.ki.5001565. PMid:16775600.

Patrakka, J., \& Tryggvason, K. (2009). New insights into the role of podocytes in proteinuria. Nature Reviews. Nephrology, 5(8), 463468. http://dx.doi.org/10.1038/nrneph.2009.108. PMid:19581907.

Pedraza-Chaverrí, J., Gonzalez-Orozco, A. E., Maldonado, P. D., Barrera, D., Medina-Campos, O. N., \& Hernandez-Pando, R. (2003). Diallyl disulfide ameliorates gentamicin-induced oxidative stress and nephropathy in rats. European Journal of Pharmacology, 473(1), 71-78. http://dx.doi.org/10.1016/S0014-2999(03)01948-4. PMid:12877940.

Pedruzzi, L. M., Stockler-Pinto, M. B., Leite, M. Jr, \& Mafra, D. (2012). Nrf2-keap1 system versus NF-kappaB: the good and the evil in chronic kidney disease? Biochimie, 94(12), 2461-2466. http://dx.doi. org/10.1016/j.biochi.2012.07.015. PMid:22874821.

Shin, I. S., Hong, J., Jeon, C. M., Shin, N. R., Kwon, O. K., Kim, H. S., Kim, J. C., Oh, S. R., \& Ahn, K. S. (2013). Diallyl-disulfide, an organosulfur compound of garlic, attenuates airway inflammation via activation of the Nrf-2/HO-1 pathway and NF-kappaB suppression. Food and Chemical Toxicology, 62, 506-513. http://dx.doi.org/10.1016/j. fct.2013.09.012. PMid:24051194.

Su, Y., Ma, X., Jiang, N., Zhang, Q., Li, M., Li, Y., \& Li, S. (2018). Toxic target of trans-crotonaldehyde in mitochondria altered by diallyl disulfides for anti-myocardial ischemia. Spectrochimica Acta. Part A: Molecular and Biomolecular Spectroscopy, 205, 568-573. http:// dx.doi.org/10.1016/j.saa.2018.07.058. PMid:30075437.

Tsai, P. Y., Ka, S. M., Chao, T. K., Chang, J. M., Lin, S. H., Li, C. Y., Kuo, M. T., Chen, P., \& Chen, A. (2011). Antroquinonol reduces oxidative stress by enhancing the Nrf2 signaling pathway and inhibits inflammation and sclerosis in focal segmental glomerulosclerosis mice. Free Radical Biology \& Medicine, 50(11), 1503-1516. http:// dx.doi.org/10.1016/j.freeradbiomed.2011.02.029. PMid:21376112.

Venkatesan, N., Punithavathi, D., \& Arumugam, V. (2000). Curcumin prevents adriamycin nephrotoxicity in rats. British Journal of Pharmacology, 129(2), 231-234. http://dx.doi.org/10.1038/ sj.bjp.0703067. PMid:10694226.

Wiggins, R. (2007). The spectrum of podocytopathies: a unifying view of glomerular diseases. Kidney International, 71(12), 1205-1214. http://dx.doi.org/10.1038/sj.ki.5002222. PMid:17410103.

Wongmekiat, O., \& Thamprasert, K. (2005). Investigating the protective effects of aged garlic extract on cyclosporin-induced nephrotoxicity in rats. Fundamental \& Clinical Pharmacology, 19(5), 555-562. http:// dx.doi.org/10.1111/j.1472-8206.2005.00361.x. PMid:16176334.

Wu, C. C., Sheen, L. Y., Chen, H. W., Tsai, S. J., \& Lii, C. K. (2001). Effects of organosulfur compounds from garlic oil on the antioxidation system in rat liver and red blood cells. Food and Chemical Toxicology, 39(6), 563-569. http://dx.doi.org/10.1016/S0278-6915(00)00171-X. PMid:11346486.

Yang, S.-M., Chan, Y.-L., Hua, K.-F., Chang, J.-M., Chen, H.-L., Tsai, Y.J., Hsu, Y.-J., Chao, L. K., Feng-Ling, Y., Tsai, Y.-L., Wu, S. H., Wang, Y. F., Tsai, C. L., Chen, A., \& Ka, S. M. (2014). Osthole improves an accelerated focal segmental glomerulosclerosis model in the early stage by activating the Nrf2 antioxidant pathway and subsequently inhibiting NF- $\mathrm{kB}$-mediated COX-2 expression and apoptosis. Free Radical Biology \& Medicine, 73, 260-269. PMid:24858719.

Yang, S.-M., Hua, K.-F., Lin, Y.-C., Chen, A., Chang, J.-M., Kuoping Chao, L., Ho, C.-L., \& Ka, S.-M. (2013). Citral is renoprotective for focal segmental glomerulosclerosis by inhibiting oxidative stress and apoptosis and activating Nrf2 pathway in mice. PLoS One, 8(9), e74871. http://dx.doi.org/10.1371/journal.pone.0074871. PMid:24069362. 